\title{
The Influence of Basic Reproduction Number on Pattern Formations in a Spatial Epidemic Model with The Susceptible Cross-diffusion
}

\author{
A. Triska ${ }^{*}$, A. Y. Gunawan, N. Nuraini \\ Departement of Mathematics, Faculty of Mathematics and Natural Sciences \\ Institut Teknologi Bandung, Jalan Ganesha 10 Bandung 40132, Indonesia
}

\begin{abstract}
In this paper, we considered a spatial epidemic model with cross-diffusion of the susceptible. The cross-diffusion of the susceptible represents the tendency of susceptible to move away from the infected. We focused on the influence of basic reproduction numbers on the pattern formations of the model through Turing analysis. The Turing space in the parameter domain was given. From numerical simulation results, the model exhibited the following patterns, i.e., the holes, holes-stripes, stripes, spots-stripes, and spots. The holes indicated the occurrence of an outbreak in a region, whereas the spots indicated otherwise. If the moderate outbreak occurred in a region, the susceptible cross-diffusion term could change the patterns which indicated that it might prevent the disease dispersion to wider areas although the basic reproduction number was greater than its threshold. However, once the outbreak occurred spatially, the action of the susceptible to move away from the infected could not prevent the outbreak anymore. The results showed that the basic reproduction number has a big influence on the pattern formations when the values of the susceptible cross-diffusion coefficient were small.

Keywords: Spatial epidemic model, cross-diffusion of the susceptible, basic reproduction number, pattern formation, Turing bifurcation.
\end{abstract}

DOI: $10.7176 / \mathrm{MTM} / 11-4-02$

Publication date:September $30^{\text {th }} 2021$

\section{Introduction}

In the last decade, spatial dependence and dispersal individuals were considered in the epidemic models since people were distributed in space and interacted with each other in their spatial neighborhoods. This model was known as the spatial epidemic model. In a spatial epidemic model, the dynamics of the disease transmission were observed through pattern formations by using Turing analysis. Alan Turing [1] described a revolutionary idea that in a reaction-diffusion system, a homogeneous steady state which is stable to small temporal perturbation under the absence of diffusion becomes unstable in the presence of diffusion term.

The spatial epidemic model with self-diffusion is an important scientific discipline to understand the effect of spatial heterogeneity in disease transmission [2]. Some researcher addressed their study with this model. Liu et al. [3] studied the effect of the diffusion term on the spatial epidemic model through Turing mechanisms. By modifying the model in [3], Sun et al. [4] studied a spatial epidemic model with a nonlinear incidence rate. Their model revealed not only the spots-stripes and stripes but also the spots. Cai and Wang [5] investigated the complex dynamics of the SIR spatial model with a nonlinear incidence rate of saturated mass action. The model of this study exhibited the patterns that grew to the spots, spots-stripes, stripes, stripes-holes, and holes. The results indicated that the speed of disease deployment was getting bigger with the increasing diffusion of infection [5]. The readers can also find other spatial epidemic models with self-diffusion in [6,7].

Wang et al. [2] modified an epidemic model of Berezovky et al. [8] into a spatial epidemic model by employing self-diffusion to represent the susceptible and infected population movement corresponding to the model in [8]. Berezovky et al. introduced an epidemic model by adding the disease-induced mortality, and emigration into the model of Kermak and McKendrick [9]. Berezovky et al. re-scaled the model so that the basic demographic reproductive number and basic reproduction number existed explicitly in the model. Wang et al. [2] analyzed the pattern dynamics due to the variation of those parameters from a spatial epidemic model with selfdiffusion.

However, in nature, there is a tendency of the susceptible to move away from the infected since they have the ability to recognize the infected and keep away from them. As consequently, Sun et al. studied the spatial pattern of the epidemic model by considering cross-diffusion of the susceptible [10]. Their results showed that the model exhibited the stripes and stripes-spots. Moreover, the qualitative analysis of the model in [10] was studied in [11]. Li et al. [12] also applied cross-diffusion of the susceptible to the spatial epidemic model in [7] and found the patterns of the spots, spots-stripes, and stripes.

It is well known that the basic reproduction number has been identified as a parameter threshold of the transmission of infectious diseases. This study is interested in analyzing the influence of basic reproduction numbers on the pattern dynamics of a spatial epidemic model with the susceptible cross-diffusion. Therefore, 
this study applied the cross-diffusion of the susceptible to the spatial epidemic model in [2]. This study is organized as follows. A spatial epidemic model with self-diffusion and cross-diffusion of the susceptible was constructed in Sec. 2. In Sec. 3, Turing analysis were carried out and the Turing space was given. In Sec. 4, the results of a series of numerical simulations and their interpretation from the biological point of view were performed. Finally, we gave briefly conclusions in the last section.

\section{The Model}

Considering the epidemic model introduced by Berezovky et al. [8], the classic model of Kermak and McKendrick [9] was modified by adding the disease-induced mortality and emigration. The total population $(N=S+I)$ was divided into two groups, susceptible $(S)$ and infected $(I)$ as follows

$$
\begin{aligned}
& \frac{d S}{d t}=r N\left(1-\frac{N}{K}\right)-\beta \frac{S I}{N}-(\mu+m) S, \\
& \frac{d I}{d t}=\beta \frac{S I}{N}-(\mu+d) I,
\end{aligned}
$$

where the reproduction of susceptible follows a logistic equation with the intrinsic growth rate $r$. Here, $K$ was the carrying capacity while $\beta$ denoted the constant infection rate. Parameters $\mu, d$, and $m$ denoted the natural mortality, disease-induced mortality, and susceptible emigration rate per capita, respectively. Note that the growth rate of susceptible was proportional to the total number of infected and susceptible so that the infected (as well as the susceptible) produced healthy susceptible offspring [8].

In the absence of the disease $(I=0)$, Model (1.1) became to the demographic equation

$$
\frac{d N}{d t}=r N\left(1-\frac{N}{K}\right)-(\mu+m) N,
$$

which led to the concept of the basic demographic reproductive number $R_{d}$, which was given by

$$
R_{d}=\frac{r}{\mu+m} .
$$

If $R_{d}>1$ then the population grew, while $R_{d} \leq 1$ implied that the population did not survive. Meanwhile, the epidemic threshold known as the basic reproductive number could be obtained by using the next-generation method that yielded

$$
R_{0}=\frac{\beta}{\mu+d} .
$$

Usually, the disease would successfully invade when $R_{0}>1$ but would die out if $R_{0} \leq 1$. More details were explained in Section 2 in [8].

Now, based on the introduction above, considering the Model (1.1) with self-diffusion and crossdiffusion of the susceptible as follows

$$
\begin{aligned}
& \frac{\partial S}{\partial t}=r N\left(1-\frac{N}{K}\right)-\beta \frac{S I}{N}-(\mu+m) S+D_{1} \nabla^{2} S+D_{3} \nabla^{2} I, \\
& \frac{\partial I}{\partial t}=\beta \frac{S I}{N}-(\mu+d) I+D_{2} \nabla^{2} I,
\end{aligned}
$$

where $D_{1}$ and $D_{2}$ were self-diffusion coefficients, while $D_{3}$ was the cross-diffusion coefficient of the susceptible. The notation $\nabla^{2}=\frac{\partial^{2}}{\partial x^{2}}+\frac{\partial^{2}}{\partial y^{2}}$ denoted the Laplacian operator in two-dimensional spaces which were used to describe the Brownian random motion. All diffusion coefficients were taken to be positive. Model (1.5) was to be analyzed under the following positive initial conditions

$$
S(\mathbf{r}, 0)>0, \quad I(\mathbf{r}, 0)>0, \quad \mathbf{r}=(x, y) \in \Omega=[0, L] \times[0, L],
$$

and the zero-flux boundary condition

$$
\frac{\partial S}{\partial \mathbf{n}}=\frac{\partial I}{\partial \mathbf{n}}=0
$$

In the boundary conditions, $\mathbf{n}$ was the outward unit normal vector of the smooth boundary $\partial \Omega$. The zero-flux boundary conditions implied that there was no incoming or outgoing population movement across the boundary.

For simplicity, let us transform the Model (1.5) into a dimensionless form by assuming that

and yield the following

$$
S^{*}=\frac{S}{K}, I^{*}=\frac{I}{K}, t=\frac{(\mu+d) t^{*}}{L^{2}}, x^{*}=\frac{x}{L}, y^{*}=\frac{y}{L}
$$




$$
\begin{aligned}
& \frac{\partial S}{\partial t}=v R_{d}(S+I)\left(1-(S+I)-R_{0} \frac{S I}{S+I}-v S+d_{1} \nabla^{2} S+d_{3} \nabla^{2} I,\right. \\
& \frac{\partial I}{\partial t}=R_{0} \frac{S I}{S+I}-I+d_{2} \nabla^{2} I,
\end{aligned}
$$

with

$$
v=\frac{\mu+m}{\mu+d}, d_{1}=\frac{D_{1}}{(\mu+d) L^{2}}, d_{2}=\frac{D_{2}}{(\mu+d) L^{2}}, d_{3}=\frac{D_{3}}{(\mu+d) L^{2}},
$$

where $v$ was defined by the ratio of the average life-span of susceptible to that of infection [8].

\section{Turing Analysis}

Turing instability is a phenomenon in which a stable solution of a non-spatial system becomes unstable when spatially heterogeneous intervention is considered. Hence, the first step in studying Turing instability is to determine the condition for an asymptotically stable equilibrium of a non-spatial system. By setting the righthand side of Model (1.8) equal to zero yields two equilibrium points, namely a diseases-free $E_{0}=\left(\frac{R_{d}-1}{R_{d}}, 0\right)$ and an endemic equilibrium $E_{1}=\left(S^{*}, I^{*}\right)$ with

$$
S^{*}=\frac{v R_{d} R_{0}-R_{0}+1-v}{v R_{0}^{2} R_{d}} \text {, and } I^{*}=\left(R_{0}-1\right) S^{*} .
$$

The variables $S$ and $I$ denote the densities of the susceptible and infected, so that only the non-negative equilibrium points have biological meaning and considered in this study. The condition for $E_{0}$ to exist from a biological point of view is $R_{d}>1$. It means that the population only grows if the basic demographic reproductive number is bigger than one so that the population has the ability to grow. Meanwhile, the conditions for $E_{1}$ to be positive are $R_{d}>\frac{R_{0}-1+v}{v R_{0}}$ and $R_{0}>1$. This study aims to analyze the Turing pattern because of the competition of $R_{0}>1$ and $d_{3}$ so that the focus is only given to the situation that the infected exist, namely $E_{1}$.

The endemic equilibrium $E_{1}$ is asymptotically stable if the trace of the Jacobian matrix that is evaluated at $E_{1}$ is negative while the determinant is positive. Let $\mathbf{J}$ is the Jacobian matrix that evaluated at $E_{1}$,

$$
\mathrm{J}=\left(\begin{array}{cc}
-\frac{v R_{d} R_{0}+R_{0}^{2}+v R_{0}-4 R_{0}-2 v+3}{R_{0}} & -\frac{v R_{d} R_{0}-2 R_{0}-2 v+3}{R_{0}} \\
\frac{\left(R_{0}-1\right)^{2}}{R_{0}} & \frac{1}{R_{0}}-1
\end{array}\right)
$$

It is easy to check that $\operatorname{tr}(\mathbf{J})<0$ and $\operatorname{det}(\mathbf{J})>0$ which is guaranteed by the biological existence conditions of $E_{1}$. Therefore, the endemic equilibrium $E_{1}$ is asymptotically stable. Next, to obtain the conditions of the emergence of Turing instability, then $E_{1}$ must be unstable when spatial is considered. Considering a perturbation near the endemic equilibrium

$$
\begin{aligned}
& S(\mathbf{r}, t)=S^{*}+\hat{S}(\mathbf{r}, t), \\
& I(\mathbf{r}, t)=I^{*}+\hat{I}(\mathbf{r}, t),
\end{aligned}
$$

with $\hat{S}(\mathbf{r}, t)=\exp (\lambda t) \exp (i \mathbf{k} \cdot \mathbf{r})$ and $\hat{I}(\mathbf{r}, t)=\exp (\lambda t) \exp (i \mathbf{k} \cdot \mathbf{r})$. Substituting Equation (1.9) into Model (1.8) and linearizing it by neglecting higher-order terms, we obtain the following polynomial characteristic

$$
\lambda^{2}+c_{1}\left(k^{2}\right) \lambda+c_{2}\left(k^{2}\right)=0,
$$

with

$$
\begin{aligned}
& c_{1}\left(k^{2}\right)=\frac{R_{0}\left(d_{1}+d_{2}\right) k^{2}+R_{0}\left(v R_{d}+R_{0}+v-3\right)-2(v-1)}{R_{0}}, \\
& c_{2}\left(k^{2}\right)=\left(d_{1}+d_{2}\right) k^{4}+b_{1} k^{2}+b_{0}, \\
& b_{1}=\frac{\left(d_{2}+d_{3}\right) R_{0}^{2}+\left(v d_{2}\left(R_{d}+1\right)+d_{1}-4 d_{2}-2 d_{3}\right) R_{0}-(2 v-3) d_{2}-d_{1}+d_{3}}{R_{0}},
\end{aligned}
$$




$$
b_{0}=\frac{\left(R_{0}-1\right)\left(R_{0}\left(v R_{d}-1\right)-v+1\right)}{R_{0}} .
$$

To induce the Turing instability, the real part of the eigenvalue, $\operatorname{Re}(\lambda)$, has to be greater than zero. Therefore, the Turing bifurcation occurs when

$$
\operatorname{Im}(\lambda)=0 \text {, and } \quad \operatorname{Re}(\lambda)=0, \quad \text { at } \quad k=k_{c} \neq 0 .
$$

By imposing the conditions (1.11) to (1.10), we obtain the critical value of the bifurcation parameter $d_{3}$

$$
d_{3}^{T}=\frac{-R_{0}^{2} d_{2}+\left(-d_{2}\left(R_{d}+1\right) v-d_{1}+4 d_{2} R_{0}+2 v d_{2}-2 \sqrt{R_{0} d_{1} d_{2}\left(R_{0}-1\right)\left(R_{d} R_{0} v-R_{0}-v+1\right)}+d_{1}-3 d_{2}\right.}{\left(R_{0}-1\right)^{2}},
$$

at

$$
k_{c}=\frac{\left(-d_{2}-d_{3}\right) R_{0}^{2}+\left(\left(4-\left(R_{d}+1\right) v\right) d_{2}-d_{1}+2 d_{3}\right) R_{0}+(2 v-3) d_{2}+d_{1}-d_{3}}{2 R_{0} d_{1} d_{2}} .
$$

The Turing instability occurs for $d_{3}<d_{3}^{T}$. By plotting the critical values of the bifurcation parameter $d_{3}^{T}$ and the positive existence condition of $E_{1}$ with respect to $R_{0}$, the Turing bifurcation diagram in $\left(R_{0}, d_{3}\right)$ for Model (1.8) is given in Figure (1.a). In Figure (1.a) we use the same parameter values in [2], i.e., $v=0.15, d_{1}=0.00001$, and $d_{2}=0.00025$. The positive critical line of $E_{1}$ and the Turing bifurcation curve divide the space into three domains. The steady-state of the model is stable if $R_{0}$ and $d_{3}$ are taken in domain I. In domain III, the positive equilibrium of $E_{1}$ does not exist. Meanwhile, for the parameters' value in domain II, the steady-state of Model (1.8) is unstable so that it induces Turing instability. In this domain, the patterns emerge so that it is called the Turing space.

Figure (1.b) shows the dispersion relation of the real part of eigenvalues corresponding to several values of bifurcation parameter $d_{3}$ whereas other parameters are fixed as $R_{0}=1.17, v=0.15, d_{1}=0.00001$, and $d_{2}=0.00025$. In Figure (1.b), the solid line corresponds to the critical Turing value $d_{3}^{T}=0.000401303$. If $d_{3}<d_{3}^{T}$, the Turing instability occurs which is shown by the dashed and dashed-dotted curves, while the dotted curve shows the Turing instability decays, i.e., $d_{3}>d_{3}^{T}$.
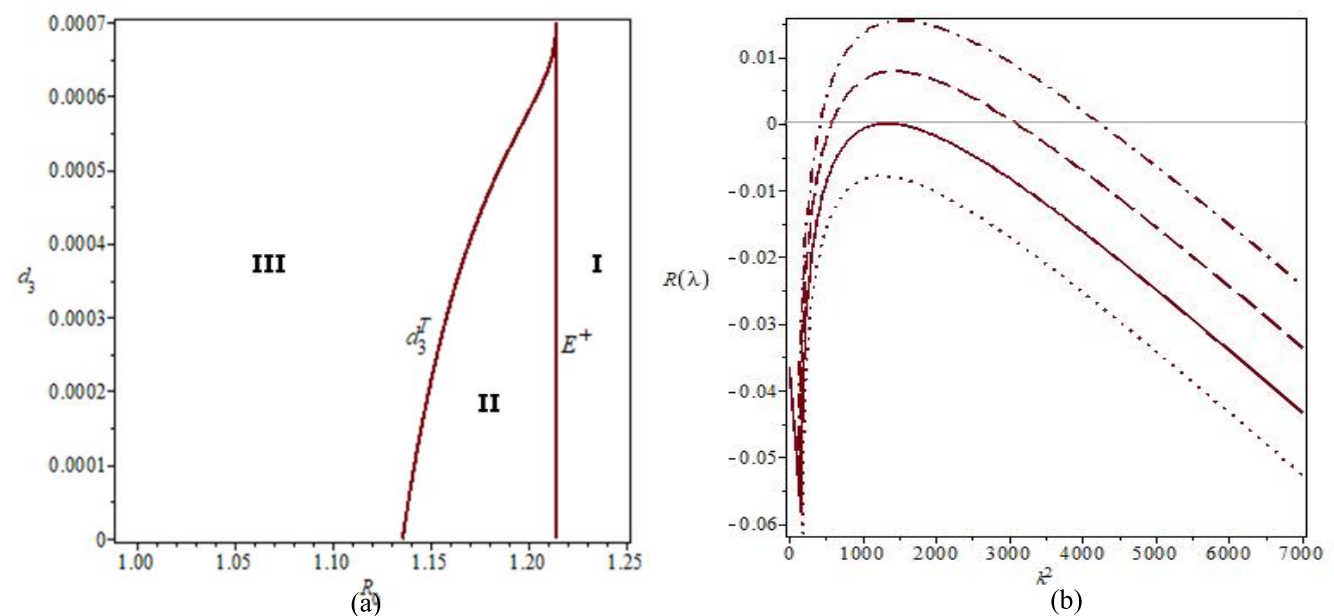

Figure 1. (a) Turing bifurcation diagram of Model (1.8) in $\left(R_{0}, d_{3}\right)$ parameters space

with $R_{d}=2, v=0.15, d_{1}=0.00001, d_{2}=0.00025$. (b) The dispersion of the real part of eigenvalues of characteristic equation (1.10) with the same value of parameters in (a) and $R_{0}=1.17$ along with $d_{3}: 0.0002$ (dashed-dotted), 0.0003 (dashed), 0.000401303 (solid), and 0.0005 (dotted).

\section{Numerical Simulations}

In this section, we perform a series of numerical simulations of Model (1.8) in two-dimensional spaces along with the qualitative results. All our numerical simulations are run with the positive initial conditions (Equation (1.6)) near the endemic equilibrium and zero-flux boundary conditions (Equation (1.7)). Our model is transformed into a dimensionless form so that the continuous problem defined by the reaction-diffusion system in two-dimensional spaces is solved in a discrete domain with the size $1 \times 1$ lattice sites. The spacing between the lattice points is defined by the lattice constant $\Delta h$. In the discrete system, the Laplacian describing diffusion is 
calculated using finite differences, i.e., the derivatives are approximated by differences over $\Delta h$. For $\Delta h \rightarrow 0$ the differences approach the derivatives [4].

The time evolution is also discrete with the steps of $\Delta t$ which is solved by using the Euler method. The Euler method approximates the concentration value at the next time step based on the change rate of the concentration at the previous time step [4]. In the present study, we take $\Delta h=0.01$ and $\Delta t=0.01$. The system is discretized in two-dimensional spaces through $x \rightarrow\left(x_{1}, x_{2}, x_{3}, \ldots, x_{N}\right)$ and $y \rightarrow\left(y_{1}, y_{2}, y_{3}, \ldots, y_{N}\right)$ with $N=100$ and other parameters are fixed as $R_{d}=2, v=0.15, d_{1}=0.00001$, and $d_{2}=0.00025$. All simulations were run until they show their characteristics and do not seem to change anymore.

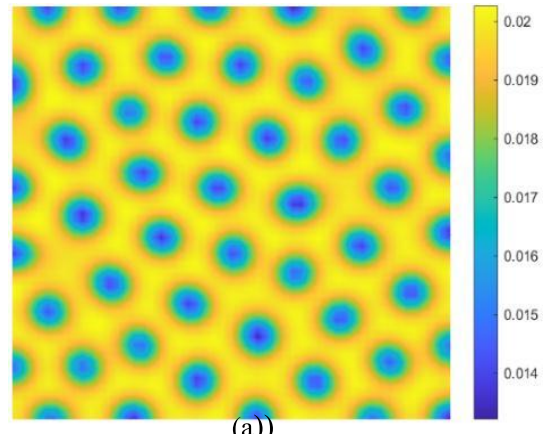

(a))

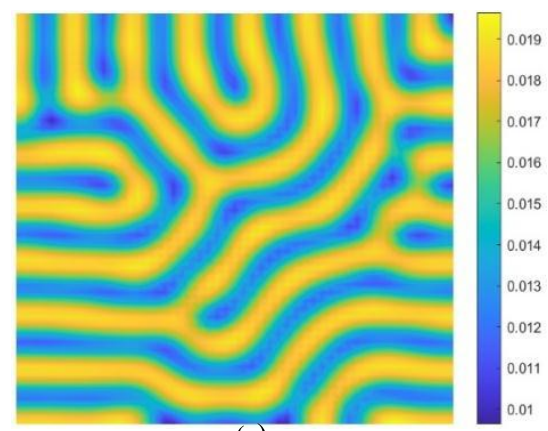

(c)

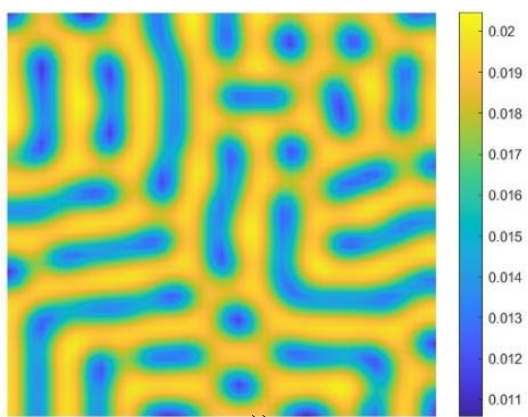

(b))

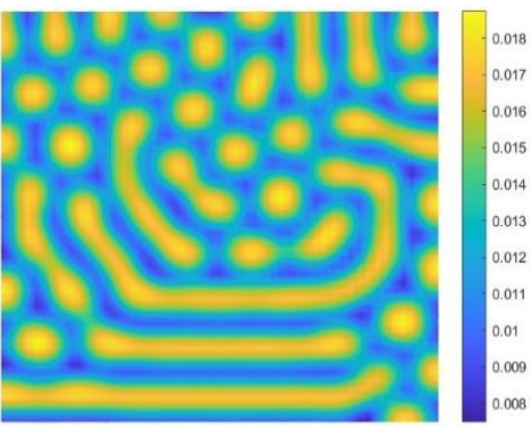

(d))

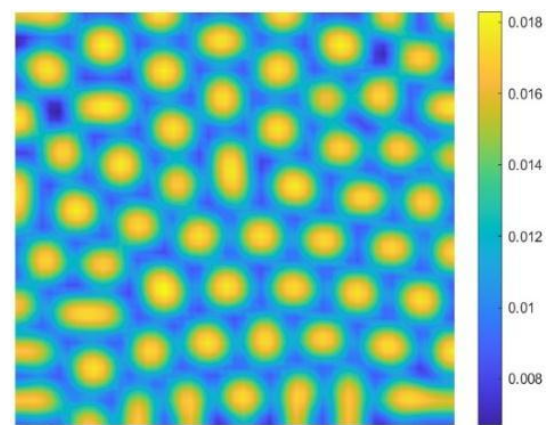

(e))

Figure 2. Turing Pattern of the infected of Model (1.8) with parameter $R_{d}=2, v=0.15$,

$$
d_{1}=0.00001, d_{2}=0.00025, d_{3}=0.00002 \text {, and } R_{0} \text { (a) } 1.14 \text {, (b) } 1.154 \text {, (c) } 1.17 \text {, (d) } 1.2 \text {, (e) } 1.213 \text {. }
$$

From the simulations, different types of patterns are observed from Model (1.8) and we found that the distributions of the susceptible and infected revealed the same type [2-7]. Since we focus on the infected distribution dynamics, only patterns of infected distribution are shown in this paper. From a series of simulations, the Model (1.8) shows five types of patterns, namely the holes, holes-stripes, stripes, stripes-spots, and spots. These patterns represent the infected distribution in a spatial domain. The holes show some areas with a low density of the infected, but the remaining region has a high density of the infected. In this situation, the outbreak may occur spatially in a region. The spots show the opposite situation, the infected with high density occurs in some areas but with low density in the remaining region. It means that the outbreak only occurs in some areas [2], not in the whole region. In Figures (3), (4), and (5), three examples of the pattern formations by time evolution are shown. 

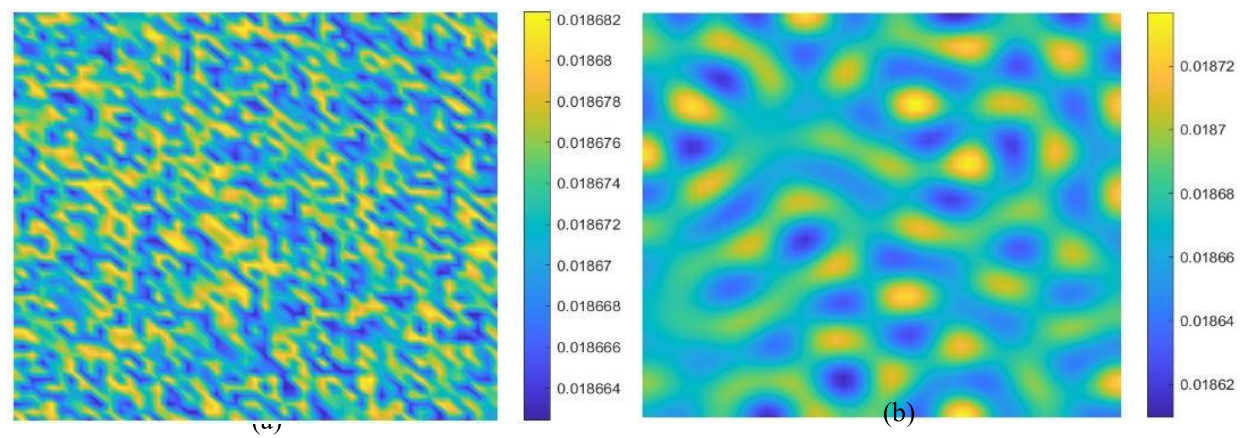

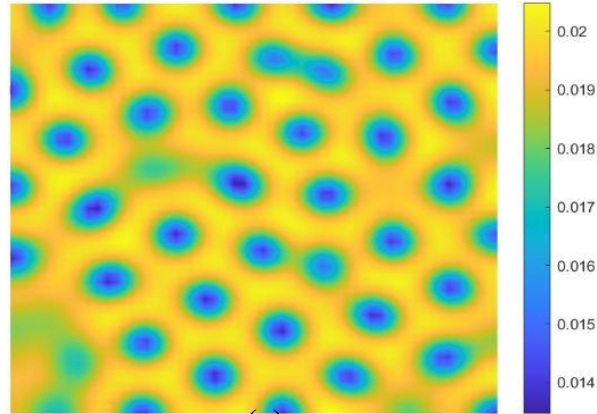

(c)

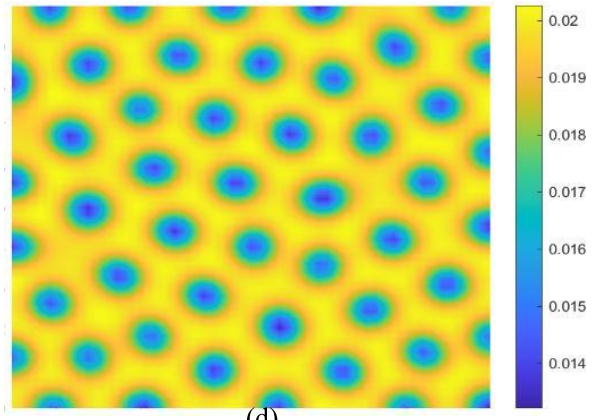

(d)

Figure 3. The holes pattern of the infected of Model (1.8) with parameter $R_{d}=2, v=0.15$,

$$
d_{1}=0.00001, d_{2}=0.00025, d_{3}=0.00002 \text {, and } R_{0}=1.14 \text {. }
$$

The model in [2] by Wang et al., i.e., without cross-diffusion of the susceptible $\left(d_{3}=0\right)$, also discovered the same types of patterns. In all our simulations, we use the same values of parameters $R_{d}, v, d_{1}$, and $d_{2}$ as in [2] since we want to study the impact of cross-diffusion of the susceptible to pattern dynamics when the $R_{0}>1$. First, we fix $d_{3}=0$ (i.e. the model with the self-diffusion only) and vary $R_{0}$ from $1.14,1.154,1.17,1.2$, to 1.213 , then we found a sequence of patterns, i.e., the holes $\rightarrow$ holes-stripes $\rightarrow$ stripes $\rightarrow$ stripes-spots $\rightarrow$ spots. We call these patterns with the aforementioned parameter values as the initial patterns.

Next, we consider cross-diffusion of the susceptible to the pattern dynamics by varying its coefficient values, $d_{3}$. An extensive simulation was carried out by taking the values of $d_{3}$ and $R_{0}$ within the Turing space and the results are shown in Figure (6). The signs $\square,{ }^{*},+, \times$, and $\square$ correspond to the holes, holes-stripes, stripes, stripes-spots, and spots, respectively.

From Figure (6), several things can be noted. The increasing of $d_{3}$ causes the Turing instability interval of $R_{0}$ narrows. Furthermore, there are interesting things that can be observed here. If the value of $d_{3}$ is fixed to be quite small (until around 0.00016) and $R_{0}$ is varied, the model produces the same sequence of patterns as the initial. However, if the value of $d_{3}$ is large enough, along with the decreasing of the Turing instability interval of $R_{0}$, it shows that the variation of $R_{0}$ produces fewer types of patterns. Moreover, when the value of $d_{3}$ exceeds 0.0003 and $R_{0}$ is varied again, then the model reveals only one type of Turing pattern, namely the spots. In this condition, it can be seen that the variation of $R_{0}$ values no longer affect the pattern. Therefore, $R_{0}$ has a big role in the pattern formations when $d_{3}$ is small. From the biological perspective, this shows that although $R_{0}>1$ and even increases, but if the value of $d_{3}$ is large enough, it can prevent the outbreak from spreading to other areas since the spots indicate that the outbreak only occurs in a certain area.

On the other hand, the increasing of $R_{0}$ causes the Turing instability interval of $d_{3}$ expands. Figure (6) also shows that the presence of cross-diffusion of the susceptible $\left(d_{3} \neq 0\right)$ may change the initial patterns and yield different types of patterns that can be seen when $d_{3}$ increases. For $R_{0}$ is equal to 1.14 and 1.15 , the initial patterns of the model are the holes. 

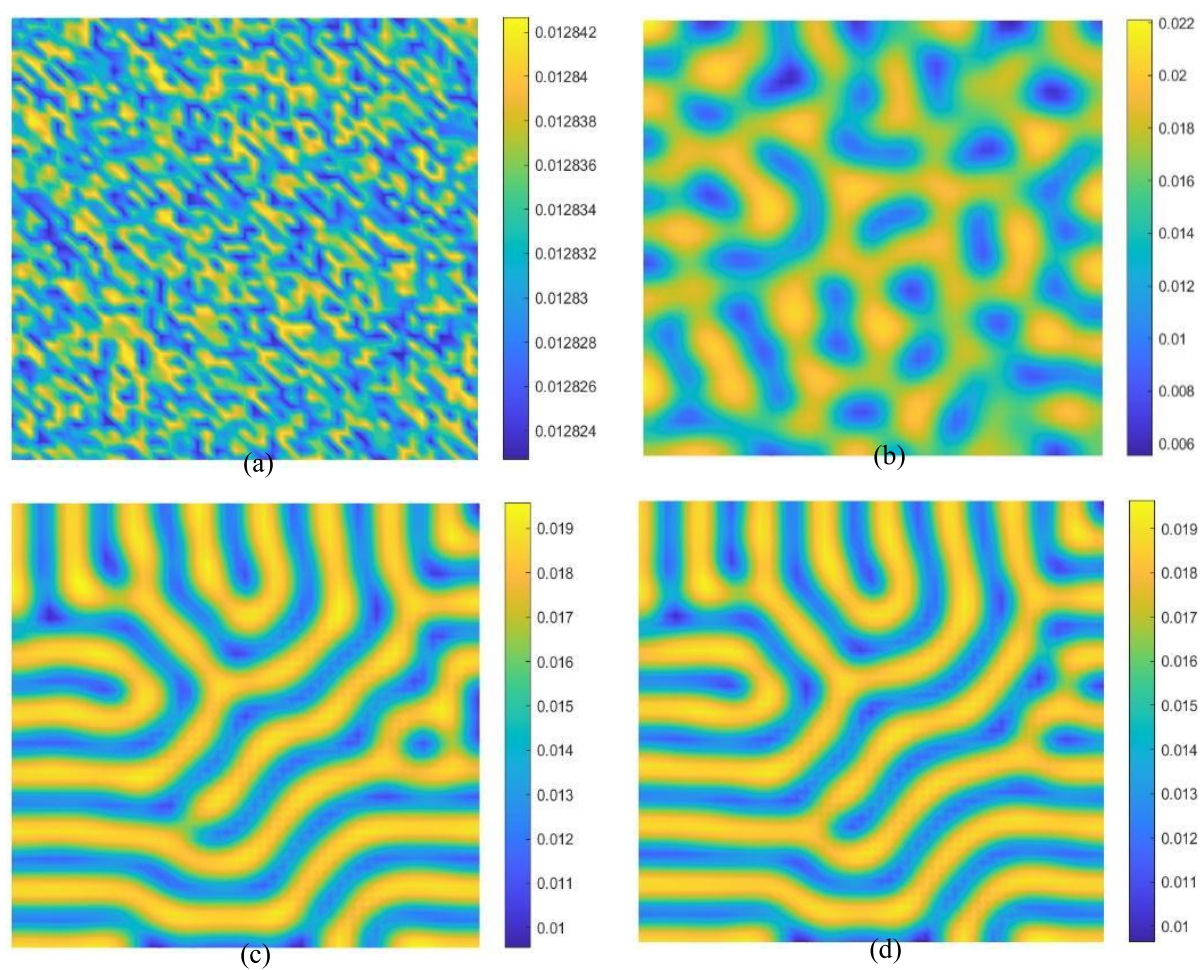

Figure 4. The stripes pattern of the infected of Model (1.8) with parameter $R_{d}=2, v=0.15$

$$
d_{1}=0.00001, d_{2}=0.00025, d_{3}=0.00002 \text {, and } R_{0}=1.17 \text {. }
$$

If the values of $d_{3}$ are increased until close to its bifurcation value (i.e., the value of $d_{3}$ for $R_{0}=1.14$ and $R_{0}=1.15$ ), the model shows the same type of pattern. The unchanged type of pattern also occurs for $R_{0}=1.154$, i.e., the initial pattern remains the same although $d_{3}$ increases until it reaches the bifurcation value. The initial pattern when the values of $R_{0}$ are 1.16,1.17, and 1.18 is the stripes. For $R_{0}=1.16$, the change of the type of pattern occurs once, i.e., the stripes $\rightarrow$ stripes-spots. Meanwhile, the increase of $d_{3}$ for $R_{0}$ is equal to 1.17 , and 1.18 results in the change of pattern types twice, i.e., the stripes $\rightarrow$ stripes-spots $\rightarrow$ spots. For $R_{0}$ are equal to 1.19 and 1.2 yield the stripes-spots initially and change into the spots during the increase of $d_{3}$. However, the unchanged type of pattern reoccurs when the model shows the spots initially for $R_{0}=1.213$. 

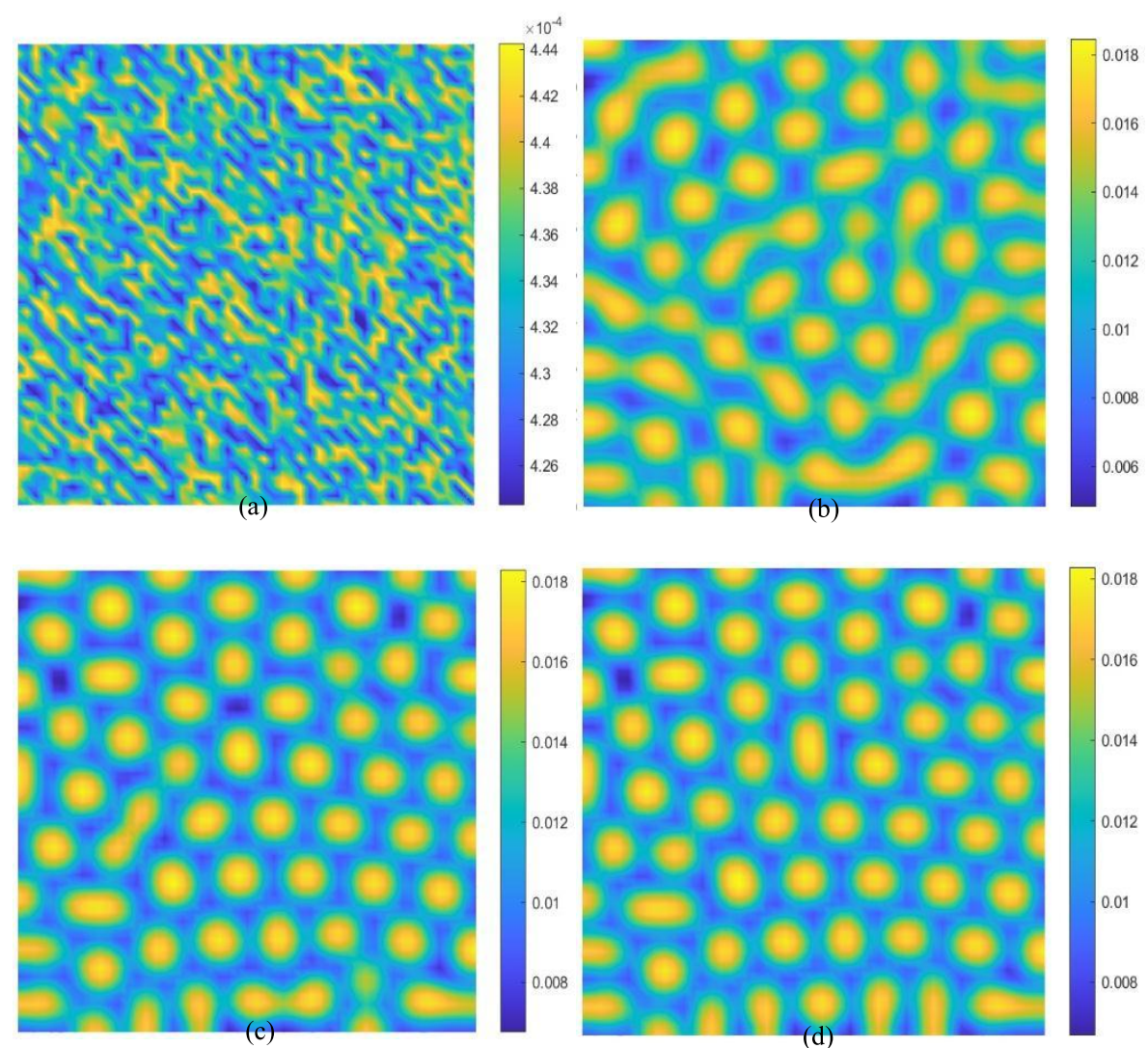

Figure 5. The spots pattern of the infected of Model (1.8) with parameter $R_{d}=2, v=0.15$,

$$
d_{1}=0.00001, d_{2}=0.00025, d_{3}=0.00002 \text {, and } R_{0}=1.213 \text {. }
$$

From an epidemiological point of view, each pattern type shows the difference of dynamics distribution of the infected. Cross-diffusion of the susceptible is able to change the density characteristic of the infected if the initial patterns are the stripes and stripes-spots. These two patterns can be interpreted as a moderate outbreak. The high density of the infected takes place only in some areas so that an outbreak does not occur in a region. Once an outbreak (or almost outbreak) is happening spatially which is represented by the holes (or the holesstripes), i.e., the high density of the infected occurs in almost all areas in a region, then the pattern does not change anymore. That is to say, people should be aware to stay away from the infected before the outbreak disperses wider.

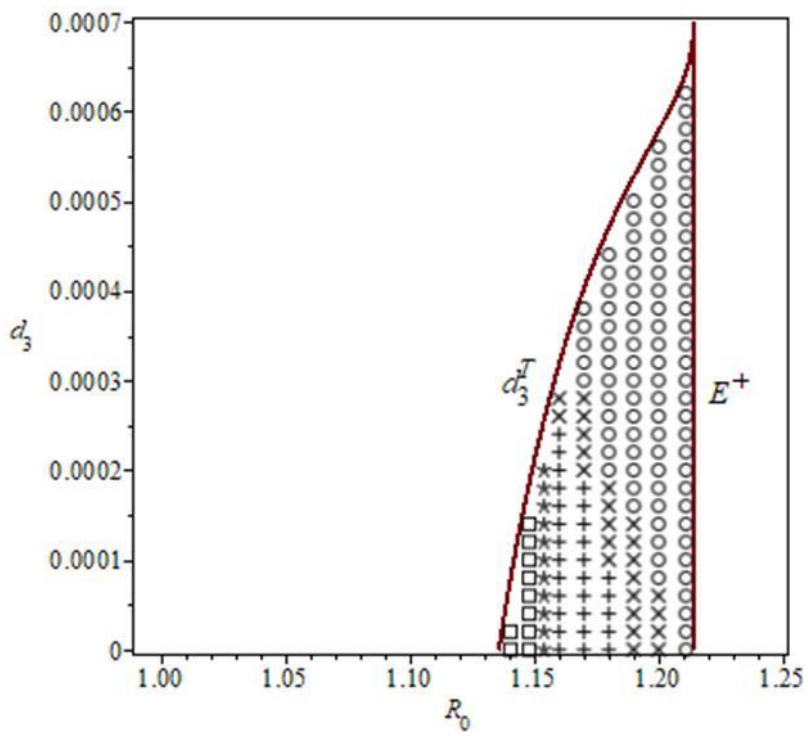

Figure 6. The emergence location of Turing Pattern of Model (1.8). The signs $\square,{ }^{*},+, \times$, and $\square$ correspond to the holes, holes-stripes, stripes, stripes-spots, and spots, respectively. 


\section{Conclusions}

In this paper, we analyzed a spatial epidemic model with self-diffusion along with cross-diffusion of the susceptible under the zero-flux boundary conditions. The value of this study lies in two aspects. First, it shows five types of Turing patterns that were obtained from numerical simulations by varying the values of basic reproduction number and the susceptible cross-diffusion coefficient within the Turing space. Second, it describes the influence of basic reproduction number and cross-diffusion of the susceptible to the Turing patterns from the epidemiological point of view.

In our numerical simulation, we found that a range of values in $\left(R_{0}, d_{3}\right)$ parameter space exists where different spatial patterns emerge. We focused on the dynamics of disease transmission with the variation of basic reproduction number and cross-diffusion of the susceptible. We observed that basic reproduction number has a big influence on the pattern formations if the speed of the susceptible to move away from the infected is low. However, the diseases' spread is getting smaller when the susceptible cross-diffusion coefficient $\left(d_{3}\right)$ increases although $R_{0}$ is getting bigger. But once the disease has spread to almost all areas in a region then the action of the susceptible to move away from the infected could not prevent the outbreak spatially anymore. In other words, our result may show that the awareness to move away from the infected must be owned since the early period of disease spreading.

\section{Acknowledgment}

This research was partially supported by the P2MI Research Grand of Institut Teknologi Bandung (62/IT1.C02/SK-TA/2021).

\section{Conflict of Interest}

The authors declare that they have no conflict of interest.

\section{References}

1. A.M. Turing, The chemical basis of morphogenesis, Phil. Trans. Roy. Soc. Lond. B 237 (641) (1952) 37-72.

2. W. Wang, Y. Cai, M. Wu, K. Wang, and Z. Li, Complex dynamics of a reaction-diffusion epidemic model," Nonlinear Analysis: Real World Applications, vol. 13, no. 5, pp. 2240-2258, 2012.

3. Q. Liu, Z. Jin, Formation of spatial patterns in an epidemic model with constant removal rate of the infectives, J. Stat. Mech. 2007 (2007) P05002.

4. G. Sun, Z. Jin, Q.X. Liu, L. Li, Pattern formation in a spatial S-I model with non-linear incidence rates, J. Stat. Mech. 2007 (2007) P11011.

5. Y. Cai, W. Wang, Spatiotemporal dynamics of a reaction-diffusion epidemic model with nonlinear incidence rate, J. Stat. Mech. (2011) P02025.

6. W. Wei-Ming, L. Hou-Ye, C. Yong-Li, and L. Zhen-Qing, Turing pattern selection in a reaction-diffusion epidemic model," Chinese Physics B, vol. 20, no. 7, p. 074702, 2011.

7. G.-Q. Sun, Pattern formation of an epidemic model with diffusion," Nonlinear Dynamics, vol. 69, no. 3, pp. 1097-1104, 2012.

8. F. Berezovsky, G. Karev, B. Song, C. Castillo-Chavez, A simple epidemic model with surprising dynamics, Math. Biosci. Eng. 2 (2005) 133-152.

9. W.O. Kermack, A.G. McKendrick, A contribution to the mathematical theory of epidemics, Proc. R. Soc. A 115 (1927) 700-721.

10. G. Sun, Z. Jin, Q.X. Liu, L. Li, Spatial pattern in an epidemic system with cross-diffusion of the susceptible, J. Biol. Sys. 17 (1) (2009) 1-12.

11. Y. Cai, D. Chi, W. Liu, and W. Wang, Stationary patterns of a cross-diffusion epidemic model," in Abstract and Applied Analysis, vol. 2013, Hindawi, 2013.

12. L. Li, J. Zhen, and S. Gui-Quan, Spatial pattern of an epidemic model with cross-diffusion," Chinese Physics Letters, vol. 25, no. 9, p. 3500, 2008. 